\title{
Et « puis » : quoi encore ? \\ Pour une nouvelle justification de la persistance de PUIS en français contemporain : le cas de Si je puis dire
}

\author{
Ciry, Guillaume \\ Laboratoire CALHISTE \\ CNRS - EA 4343 \\ Université de Valenciennes et du Hainaut Cambrésis \\ guillaume.ciry@univ-valenciennes.fr
}

\section{Introduction}

La littérature explique traditionnellement le maintien de la forme puis, première personne du verbe pouvoir, de deux manières : soit syntaxiquement, dans le cas précis de l'inversion du sujet dans puis-je (*peux-je étant impossible) soit par l'argument du registre, la forme, dans son emploi relevant alors de la langue soutenue. Il en va ainsi, par exemple, dans l'article du TLFi consacré au verbe pouvoir :

\footnotetext{
Conjug. Ind. prés.: je peux ou je puis (quand je suit le verbe toujours puis-je : puis-je vous aider ?) (...) La forme puis remplace peux, à la $1^{\text {re }}$ pers. du sing., toujours en forme interr., souvent dans la lang. soutenue à la forme affirm.
}

ou encore dans le Grand Robert de la Langue Française :

REM. 1. À la première personne du singulier de l'indicatif, la forme je puis, plus ancienne, est archaïque ou un peu affectée (sauf dans l'interrogation).... Il arrive qu'on emploie je puis pour éviter la répétition de je peux.

Nous proposons dans le présent article une troisième justification, pragmatique cette fois, qui, au même titre que les deux précédentes, viendrait expliquer le maintien de puis. En effet, la persistance de puis est particulièrement nette au sein du marqueur discursif (MD) si je puis dire, marqueur largement plus fréquent dans l'emploi en discours que sa forme similaire si je peux dire.

Dès lors, notre démarche consistera tout d'abord à proposer une approche diachronique, dans un corpus constitué à partir de plusieurs requêtes ciblées soumises à la base Frantext, pour décrire deux étapes qui ont mené à la construction du marqueur tel qu'il est employé en français contemporain. Puis, dans une perspective cette fois plus synchronique, nous proposerons une description de la fonction remplie par le phrasème, ce dernier fonctionnant effectivement et comme un connecteur textuel et comme un marqueur discursif au sens de Gaétane Dostie (Dostie 2004 : 43). Enfin, nous conclurons en abordant la question de l'existence du paradigme « large » de MD commençant par Si dans lequel puis, justement, trouve une troisième explication à la raison de son maintien en français actuel.

Pour appréhender au mieux les mécanismes dont il sera présentement question, nous nous situerons notamment dans le cadre théorique de G. Dostie. Nous nous fonderons en particulier sur la distinction qu'elle fait entre la grammaticalisation au sens de Traugott (appelée chez G. Dostie grammaticalisation1) et la pragmaticalisation (appelée chez G. Dostie grammaticalisation2) :

D'une part une unité lexicale peut développer des emplois grammaticaux; elle aura alors été soumise à un processus de "grammaticalisation ». D'autre part, une unité lexicale/grammaticale peut développer des emplois où elle ne joue pas un rôle sur le plan référentiel, mais bien sur le plan conversationnel ; elle sera alors le résultat d'un processus de «pragmaticalisation » (Dostie $2004: 27$ ) 


\section{Description diachronique des deux étapes de construction du MD Si je puis dire}

\subsection{La constitution des corpus : requêtes, résultats \& commentaires}

Pour notre étude, nous avons tout d'abord choisi de soumettre à l'ensemble des textes de la base Frantext une première série de requêtes ${ }^{1}$ pour « cadrer » au mieux l'analyse des étapes de construction du MD. Nous indiquons ci-après et par ordre décroissant de fréquence, les termes des requêtes en question, le nombre de résultats obtenus ainsi que les dates des occurrences respectivement les plus anciennes et les plus récentes :
A. Requête si je puis : 1711 résultats entre 1515 et 2011
B. Requête si je puis dire : 397 résultats entre 1659 et 2008
C. Requête si je puis m'exprimer ainsi : 87 résultats entre 1731 et 1982
D. Requête si je puis parler ainsi : 58 résultats entre 1713 et 1982
E. Requête si je puis me permettre : 20 résultats entre 1776 et 2006
F. Requête si je puis ainsi parler : 14 résultats entre 1824 et 1936
G. Requête si je puis ainsi m'exprimer : 6 résultats entre 1824 et 2004

Les requêtes supra ont été soumises à la base avec deux objectifs :

i. Premièrement nous souhaitions isoler dans un ensemble large (ie requête A. si je puis) la part que représente la forme grammaticalisée ou le MD (requête B. si je puis dire) afin de mesurer son taux de fréquence et donc son importance. Par suite, nous constatons que si je puis dire représente près d'un quart (un peu plus de $23 \%$ ) de la totalité des expressions contenant les unités $s i+j e+p u i s$, ce qui est le signe d'une implantation significative de la forme (grammaticalisée ou MD) y compris dans un corpus écrit ${ }^{2}$.

ii. Deuxièmement, nous voulions mesurer la prédominance de la forme grammaticalisée ou du MD Si je puis dire par rapport à d'autres $\mathrm{MD}$ ou formes grammaticalisées ressemblant(e)s car mêmement construit(e)s avec puis. Nous remarquons en effet que si nous additionnons tous les résultats de ces formes ressemblantes (ie les résultats des requêtes $\mathrm{C}$. à $\mathrm{G}$ supra) nous sommes très loin du résultat de la requête $\mathrm{B}$. si je puis dire (la somme des requêtes $\mathrm{C}$. à $\mathrm{G}$. est de 185 résultats contre 397 pour la seule requête B.). Les données comparatives que nous venons d'évoquer, dans le détail, nous permettront donc de décrire précisément le chemin par lequel les éléments composant la forme grammaticalisée ou le MD sont passés pour aboutir à la forme que nous connaissons aujourd'hui.

\subsection{Si je puis dire : un MD constitué en deux étapes successives}

\subsubsection{Première étape : Si je puis}

Tel que nous le connaissons actuellement, et tel que nous en décrirons les mécanismes infra en 3, le MD qui nous intéresse existe donc sous la forme si je puis dire. Or, en rentrant dans le détail du corpus constitué des requêtes indiquées supra, et en particulier en considérant les résultats de la seule requête A, nous avons relevé plusieurs cas de grammaticalisation voire de pragmaticalisation de la suite $s i+j e+p u i s$ comme dans (1), (2), (3), (4) \& (5) :

(1) Il faut (si je puis) qu'en ce monde Goliath, qui ailleurs ne fonde Son espoir, surmonte, et prospere :

Et qu'ainsi tousjours il espere (Des Masures, Louis, David combattant, 1566)

(2) Le bélier? il faut, si je puis, L'écorner en la mesme place ; Et vaut mieux qu'en passant je face Semblant de ne les aviser. (Baïf, Jean-Antoine, Le Brave, 1573) 
(3) Mais il n'adviendra point, si je puis, comme il pense

Car je luy bailleray premier la recompense

Qu'ont mérité ses faicts (La Taille, Jean de, Alexandre, 1573) ${ }^{3}$

(4) Ne tourne ja la truie au fond. Car, si je puis, tu ne prendras plus lievres avec ces levriers. (Lavardin, Jean de, La Celestine, 1578)

(5) Denis Vous avez menti bien humblement

Hesiode C'est vous, si je puis

Denis Mais bien vous, sans vous faire tort.

(Beroalde de Verville, François, Le Moyen de parvenir, 1610)

Pour la description et l'analyse de ces cinq exemples, nous considérons que le cas le plus pragmaticalisé et le plus illocutoire est $(5)^{4}$ puisqu'il fait clairement référence à une attitude d'opposition sur l'auteur du mensonge. De ce fait, nous pouvons clairement établir que cet exemple place si je puis au-delà de la grammaticalisation, le phrasème s'étant effectivement pragmaticalisé. En ce sens, si je puis fonctionne ici comme une adresse à un interlocuteur afin de lui opposer une fin de non recevoir et d'interrompre le mécanisme de réception par accord tacite. Nous pourrions ainsi gloser cet emploi de si je puis pragmaticalisé par Si je puis me permettre (de ne pas vous laisser dire/tenir de tels propos). Nous remarquerons à ce titre qu'une voie de pragmaticalisation existe avec si je puis me permettre (voir requête E. supra et 3.3.2 infra pour la description).

Les exemples $(1)^{5}$ à $(4)^{6}$ sont sans doute plus ambigus dans la mesure où ils peuvent donner lieu à deux interprétations :

i. Ils peuvent premièrement être considérés comme fonctionnant au sein de l'énoncé. En (1), la possibilité concernerait le fait que Goliath prospère, en (2) le fait d'écorner le bélier, en (3) le fait que les plans du tiers évoqué ne se dérouleront pas comme ce dernier l'a prévu et en (4) le fait de faire en sorte qu'avec ces lévriers l'interlocutrice n'attrapera plus de lièvres. Dans les quatre cas, il s'agit pour celui qui le dit (les quatre exemples sont en effet tirés de pièces de théâtre, voir notes $2 \& 4$ infra), d'évoquer la condition de son pouvoir dans la réalisation d'un procès. Dans ce cas, il fonctionnerait comme un emphatiseur ou intensifieur ${ }^{7}$.

ii. Dans le même temps, ces quatre mêmes exemples peuvent être interprétés comme ne portant cette fois plus sur un procès contenu dans l'énoncé mais plutôt sur une attitude par rapport au locuteur, dans la situation d'énonciation. Dans ce cas, il pourrait être légitimement considéré comme un marqueur d'écoute au sens de G. Dostie (Dostie 2004 : 48) et ainsi relever de la catégorie des marqueurs discursifs à part entière :

Ils [les marqueurs d'écoute] permettent au coénonciateur de manifester son engagement dans l'échange en cours. Cet engagement peut être d'une intensité variable (accusé de réception, support, relance), d'orientation positive (accord, approbation) ou négative (désaccord, réticence).

A l'échelle illocutoire, nous pouvons effectivement réinterpréter (1) et (2) sur le plan conversationnel comme une permission demandée par le locuteur au colocuteur pour énoncer une obligation tout en signalant l'importance de la chose dite à celui ou à celle à qui le conseil est adressé et en (3) et (4) comme une permission que le locuteur s'accorde pour donner un conseil profitable à son colocuteur. En ce sens, si je puis MD aurait les caractéristiques de la fonction conative du langage dont l'action principale sur le récepteur serait de favoriser une écoute diligente. Il ne s'agit alors plus d'emphatiser un terme contenu \& identifiable au sein de l'énoncé mais d'attirer l'attention de l'interlocuteur.

Cette première partie nous aura donc permis de mettre en relief l'existence, tôt dans la chronologie des résultats, d'un premier MD construit autour des trois seuls éléments $s i+j e+$ puis. Il convient dès lors de s'interroger sur la deuxième étape dans la constitution du MD tel que nous le connaissons, ie l'agrégation non plus de trois mais de quatre éléments : $(s i+j e+p u i s)+$ dire. 


\subsubsection{De si je puis à si je puis dire}

La première occurrence de si je puis dire relevée dans notre corpus à partir de la requête $\mathrm{B}$ date de 1659 et est relevée dans un texte de Robert Arnauld D’Andilly, Le Chemin de la perfection :

(6) Moins nous avons, moins j'ay de soin : et nostre seigneur sçait qu'il est tres-vray que la nécessité ne me donne pas tant de peine que l'abondance, si je puis dire avoir éprouvé de la nécessité, vû la promtitude avec laquelle il a toûjours plû à Dieu de nous secourir. (Robert Arnauld D'Andilly, Le Chemin de la perfection, 1659)

Nous noterons d'emblée que (6) n'est interprétable ni en emploi grammatical ni en emploi discursif puisqu'il est syntaxiquement lié. Il faudra attendre plus d'un siècle pour voir apparaître dans nos résultats la première occurrence de si je puis dire grammaticalisé, en (7) :

(7) A la vérité, Descartes a soumis ces principes impalpables, et, si je puis dire, cette poussière métaphysique, aux lois d'une géométrie ingénieuse (Henri Bernardin de Saint-Pierre, Etudes de la nature, 1784)

L'emploi ici est interprétable sur les deux plans décrits supra pour l'étape si je puis, à savoir :

i. L'interprétation peut être effectuée à l'échelle de l'énoncé et être cataphorique. d'introduire une reformulation emphatisée au sein de l'énoncé, si je puis dire marquant classiquement l'introduction d'une tournure à venir, portant sur un terme qui reste dans l'énoncé. Dans ce cas, et dans le cadre théorique décrit supra page 1, si je puis dire serait grammaticalisé (ie non pragmaticalisé), c'està-dire à l'étape grammaticalisation1 selon les termes de Dostie (Dostie 2004 : 29)

ii. L'interprétation peut être faite au-delà de l'énoncé, ie dans la situation d'énonciation, tout en restant cataphorique. Dans ce cas, le locuteur attire l'attention sur l'originalité du terme à suivre au regard des sémantismes a priori opposés des deux termes associés (unité concrète et minuscule pour poussière vs concept relevant de l'abstraction philosophique au sens «large » pour métaphysique) mais également au regard du contexte, en l'occurrence l'évocation de Descartes, autorité intellectuelle s'il en est, à qui l'on attribue des préoccupations aussi incongrues que celle du traitement de la poussière. Dès lors, si je puis dire n'est plus l'outil de la portée dans l'énoncé mais est un marqueur d'écoute au sens défini supra. Dans cette configuration, il peut être considéré comme un $\mathrm{MD}$, dans la sphère pragmatique, à l'étape grammaticalisation2 au sens de Dostie (Dostie 2004 : 29).

Pour être complet sur les premières occurrences de si je puis dire, nous soulignerons le fait qu'elles proviennent du même auteur, en l'occurrence Bernardin de Saint-Pierre : les deux premières datent de 1784 (tome 2 des Etudes de la nature), les trois suivantes datent de 1814 (tome 2 des Harmonies de la nature). Ce point mérite d'être signalé car, dans les deux interprétations que nous venons de mentionner, si je puis dire a un rôle d'intensifieur dans l'énoncé ou attentionnel lorsqu'il est MD, rôle évidemment facilité par la présence dans le phrasème du modal puis. En effet, soit il intensifie, dans l'énoncé, l'expression «poussière de métaphysique", soit, dans la situation d'énonciation, il sert à attirer l'attention sur l'audace sémantique (ou «cultuelle» au regard de l'importance de Descartes) à laquelle l'auteur se livre et qu'il revendique comme telle, comme nous venons de le voir. Ainsi, dans le cas de la première interprétation, si je puis dire serait, dans la chaîne de grammaticalisation, dans la phase de subjectivation telle que synthétiquement définie par C. Marchello-Nizia (Marchello-Nizia $2006: 26$ ) :

En tant que notion sémantico-pragmatique, ce terme désigne le fait que le locuteur rende son discours plus expressif pour agir sur l'allocutaire. Il renvoie au processus de communication. C'est en ce sens qu'on peut comprendre le 'désir d'expressivité' évoqué déjà par Meillet (1912) comme étant à la source des grammaticalisations.

Dès lors, même si une ambiguïté demeure sur l'interprétation que l'on peut donner de si je puis dire, grammaticalisé ou pragmaticalisé au point d'être un MD, il n'en reste pas moins que l'analyse qui résiste à cette ambiguïté est celle du phénomène de subjectivation et ce dernier est bien le signe que la forme est de toute façon et au minimum grammaticalisée dès les premiers relevés et que le rôle de cette forme est à 
rechercher du côté d'un certain «désir d'expressivité » explicitement assumé par l'énonciateur, dans l'énoncé certainement, dans la situation d'énonciation peut-être. ${ }^{9}$

Suivant logiquement ce processus, si je puis dire traverse ainsi le dix-neuvième siècle (18 occurrences) et continue à être l'objet d'appropriations régulières en français moderne et contemporain, au point d'apparaître, de par sa récurrence, comme l'outil de manifestation de la présence du locuteur par excellence. En effet, notre corpus recense 347 occurrences pour le seul vingtième siècle et déjà 29 occurrences pour la première décennie du vingt-et-unième siècle. Ses positions et fonctions varient peu au fil du temps, les rôles et positions étant très tôt ce qu'ils sont aujourd'hui, comme il en sera question infra. Son emploi comme outil de subjectivation est confirmé, du fait qu'il est très rare que les énonciateurs ne l'utilisent qu'une seule fois. Certains se distinguent même par un emploi intensif de si je puis dire: Charles Dubos (78 occurrences), Charles Péguy (29 occurrences), Liliane Schroeder, dans son Journal d'occupation (15 occurrences), Louis Althusser (11 occurrences), Raymond Queneau (10 occurrences), Jacques Derrida (8 occurrences) ou encore Pascal Quignard (7 occurrences). Cette réappropriation par différents auteurs vient confirmer le phénomène initial de subjectivation décrit supra puisqu'elle correspond au processus de réappropriation subjective, typique pour les formes grammaticalisées voire même pragmaticalisées (Paillard $2011: 16$ ).

Nous retirons donc de cette étude diachronique deux faits majeurs pour décrire l'évolution du MD si je puis dire:

i. D'une part la fixation de position ${ }^{10}$ s'est faite autour de trois termes : $s i+j e+$ puis, l'élément sur lequel la modalité du locuteur s'exerce restant alors implicite. Par la suite, la fixation de position s'élargit autour de quatre éléments cette fois, $s i+j e+$ puis + dire, l'élément sur lequel la modalité du locuteur s'exerce devenant dès lors explicite, la nouvelle fixation de position faisant du reste l'objet d'hésitations avec d'autres combinaisons possibles (cf. requêtes $\mathrm{C}$ à $\mathrm{G}$ supra). C'est à ce stade, une fois les termes à fixer choisis, que si je puis dire est coalescent ${ }^{11}$.

ii. Si je puis dire peut être analysé comme un ensemble grammaticalisé dans le sens où il porte sur un ou plusieurs terme(s) présent(s) dans l'énoncé. Il fonctionne alors comme un intensifieur dans l'énoncé, ce rôle étant à mettre en lien avec la présence du modal puis. Dans le même temps, il peut être analysé comme un MD à part entière, le locuteur l'employant non plus à la seule échelle de l'énoncé mais à celle de son interlocuteur, de la conversation et donc de l'énonciation en tant que telle. Dans ce cas, le MD a une nette fonction attentionnelle (il n'est plus intensifieur) qui consiste à interrompre la linéarité de l'énoncé, le temps d'une parenthèse qui se situe au niveau de l'énonciation, afín de sensibiliser l'interlocuteur à l'originalité d'une formulation.

Il convient désormais de voir ce qu'il en est des rôles, des positions et de la dualité de si je puis dire en français contemporain.

\section{Description synchronique de si je puis dire en français contemporain.}

Pour mener la description analytique de si je puis dire en français actuel, nous poserons liminairement trois questions auxquelles nous répondrons dans cette partie :

1- Si je puis dire grammaticalisé et ancré dans l'énoncé joue-t-il un plutôt un rôle d'intensifieur anaphorique ${ }^{12}$ ou plutôt un rôle d'intensifieur cataphorique?

2- La dualité décrite dans l'étude diachronique (notamment la possible interprétation à l'échelle de l'énoncé et à l'échelle de l'énonciation) persiste-t-elle ou s'estompe-t-elle en français contemporain au profit d'une pragmaticalisation plus marquée et plus avancée ?

3- Alors que nous relevions supra une variabilité paradigmatique si je puis / si je puis dire, existe-til en français contemporain d'autres formes paradigmatiques avec lesquelles si je puis dire serait amené à varier?

Pour être très précis dans l'analyse synchronique que nous proposons dans cette partie, nous avons choisi de nous pencher sur les seules occurrences postérieures à l'an deux mille. Ce parti pris nous permet 
d'avoir une approche à la fois plus nette et plus aboutie (car plus contemporaine) des phénomènes. Notre choix repose également sur le fait que si je puis dire représente une part très importante de la seule requête si je puis (cf. requête $\mathrm{A}$ en 1.1) et ce dès la première moitié du dix-neuvième siècle. L'étude synchronique peut donc s'appuyer sur un ensemble certes restreint mais très représentatif car tendanciellement confirmé depuis plus d'un siècle. En effet, sur les trente-sept résultats relevés entre 2000 et 2011 pour la requête si je puis, 29 associent $s i+j e+$ puis + dire.

\subsection{Si je puis dire en français contemporain}

Ainsi, et pour apporter des éléments de réponse à la première question liminaire, notre observation des résultats de l'ensemble évoqué supra nous permet tout d'abord de préciser que le rôle d'intensifieur au sein de l'énoncé n'est pas univoque, au contraire. En effet, si je puis dire peut occuper trois positions sensiblement différentes, ces dernières pouvant agir sur l'interprétation de la forme, notamment en transformant la portée en relation et en faisant disparaître le mécanisme d'intensification au profit d'un rôle attentionnel entre deux locuteurs :

i. Intensifieur anaphorique

ii. Intensifieur cataphorique

iii. Si je puis dire ana-cataphorique ${ }^{13}$

\subsubsection{Si je puis dire intensifieur anaphorique}

Sans être exclusivement analysable dans ce mode de fonctionnement, si je puis dire a tout de même une tendance très nette à fonctionner comme un emphatiseur a posteriori, ie en anaphore. Cette tendance existe déjà aux dix-neuvième et vingtième siècles, elle se confirme largement en français contemporain comme en (8), (9) et (10) :

(8) Treize : je prends ce mot dans un sens extrêmement positif, si je puis dire. (Olivier Rolin, Tigre en papier, 2002)

(9) (...) Stéphane Roche, dont la thèse établit une série de « diarogrammes », si je puis dire, qui donnent à lire une courbe de température du journal et son évolution sur une trentaine d'années. (Philippe Lejeune, Signes de vie, le pacte autobiographique 2,2005$)^{14}$

(10) Ses bijoux cliquettent. De fil en aiguille, si je puis dire, car elle déballe en même temps avec le camériste la robe épinglée (Pierre Guyotat, Coma, 2006)

(11) Je compte sur la retraite pour reprendre les choses en main, si je puis dire : réviser les poèmes que je voudrais conserver le plus longtemps possible à ma disposition dans ma tête (Jacques Roubaud, $\mathrm{La}$ Dissolution, 2008)

Dans (8), (9) et (10), des indices visibles et indiscutables nous indiquent que si je puis dire est anaphorique, l'élément sur lequel s'exerce la portée étant effectivement signalé tantôt par la position finale dans la l'énoncé ${ }^{15}$, tantôt par un signe de ponctuation, tantôt par un enchaînement rendant inaccessible le co-texte droite. En effet, en (8), nous n'avons d'autre choix que d'interpréter l'élément sur lequel s'exerce la portée comme se trouvant à gauche puisque si je puis dire termine l'énoncé, ce qui empêche toute orientation cataphorique. En (9), l'ambiguïté est levée par le fait que la ponctuation (en l'occurrence les guillemets) vient indiquer sur quel terme porte l'emphase (la portée est « fléchée » par la ponctuation). En (10), la présence d'une conjonction de coordination vient quant à elle « saturer » le cotexte droite de si je puis dire, rendant assez improbable une portée qui concernerait une formulation à venir, la frontière établie par «car» étant sans doute aussi hermétique que celle dressée par le point en (8). Dans ce cas, au contraire, « car » sert à isoler la formulation sur laquelle porte l'emphase pour livrer des explications sur le fait d'avoir choisi d'emphatiser cette formulation en particulier (notamment au vu du contexte). 
L'exemple (11) mérite quant à lui un commentaire particulier. Au contraire des trois précédents, il ne dispose d'aucun «appui» particulier rendant indiscutable son fonctionnement anaphorique. Nous pourrions tout aussi bien le comprendre comme agissant a posteriori (en emphatisant « reprendre les choses en main ») que comme agissant a priori sur les termes à suivre (emphatisant alors : " réviser les poèmes que je voudrais conserver...»). Or, il nous paraît "naturel» de privilégier l'interprétation anaphorique en tranchant pour un référent situé à gauche et non à droite, en dépit des deux points suivant si je puis dire, qui eux-mêmes peuvent favoriser la décision cataphorique (si je puis dire ce que je m'apprête à énoncer). La décision en faveur de l'anaphore en (11) peut du reste venir poser la question des appuis syntaxique et de ponctuation décrits supra. La langue apporterait-elle des « renforts » pour que si je puis dire soit, en discours, indiscutablement anaphorique? Il convient bien évidemment de se poser cette question au regard d'une description précise des autres possibilités, notamment du fonctionnement cataphorique.

\subsubsection{Si je puis dire intensifieur cataphorique}

Comparativement, nous ne trouvons pas de fonctionnement d'emphase cataphorique sans appui systématique comme nous pouvons de trouver pour l'exemple (11) à propos de l'emphase anaphorique. L'appui n'est donc pas octroyé en faveur de l'anaphore. Au contraire, dans tous les cas où si je puis dire fonctionne indiscutablement comme un intensifieur cataphorique, le phénomène est garanti par la présence d'un « appui » pour renforcer l'orientation cataphorique comme en (12) et (13) :

(12) J'ai en cours l'édition du journal d'un adolescent du XIXe siècle - mais si je puis dire, c'est la routine, une routine passionnante (Philippe Lejeune, Signes de vie, le pacte autobiographique 2, 2005)

(13) (...) l'intertextualité ne met en rapport que l'écriture et la lecture, tandis que notre formule « écriture de soi et lecture de l'autre » évoque aussi, si je puis dire, l'idée d'interpersonnalité : à travers la construction de l'écriture, c'est le moi qui serait sous influence. (Philippe Lejeune, Signes de vie, le pacte autobiographique $2,2005)$

Les mécanismes qui permettent de trancher, pour si je puis dire, en faveur du rôle d'intensifieur cataphorique en (12) reposent sur la ponctuation, comme en (9) avec les guillemets (ici, c'est le tiret séparant du co-texte gauche qui joue le rôle de «balise ») et sur la conjonction «mais » qui réoriente le propos (comme «car» supra en (10) introduisait une explication). En (13), la saturation du co-texte gauche est assurée par «aussi » qui annonce un ajout et, immédiatement après, une emphase à venir. De fait, en ôtant ces mécanismes de délimitation, en (12) la portée de l'emphase deviendrait opaque (on se demanderait alors si l'emphase porte sur «adolescent du XIXe siècle » ou sur «c'est la routine ») et en (13), l'emphase indiscutablement cataphorique laisserait place à un mécanisme d'ana-cataphore : en ce sens, ce n'est plus « idée d'interpersonnalité » qui serait intensifié mais on attirerait plutôt l'attention sur le caractère étrange ou audacieux de l'association "évoque " + «idée d'interpersonnalité » dans l'expression «évoque l'idée d'interpersonnalité » alors qu'il n'y a a priori rien d'audacieux ou d'étrange dans cette expression. Une telle dissolution de la portée signerait sa disparition au sein de l'énoncé (elle se transformerait alors en relation comme analysé infra) et marquerait de facto le passage d'une intensification cataphorique à un mécanisme ana-cataphorique dont l'unité n'est plus entièrement dans le co-texte gauche mais se retrouve «éclatée ». C'est précisément ce dernier mécanisme que nous proposons maintenant de traiter.

\subsubsection{Si je puis dire ana-cataphorique}

Pour la clarté de notre travail d'analyse et de description, nous choisissons d'appeler ana-cataphoriques les mécanismes qui consistent à commencer à attirer l'attention sur plusieurs unités composant une expression avant même que toutes les unités en question aient été produites dans l'ordre de la chaîne parlée (ce qui les rend individuellement inintelligibles en l'état) comme en (14) et (15) 
(14) Etait-ce le livre qui faisait, si je puis dire, le tour complet de la mélancolie, ne laissant aux successeurs que la possibilité de rejouer avec d'autres notes telle ou telle «partition »? (Jean-Bertrand Pontalis, Traversée des ombres, 2003)

(15) Eh bien Jordane vérifie l'hypothèse, puisqu'il prend sa source, si je puis dire, au flanc de ma première fiction et qu'il en découle naturellement. (Jean-Benoît Puech, Présence de Jordane, 2002)

Ainsi, si je puis dire interrompt à « faisait » l'expression « faisait le tour complet de la mélancolie » et, en (14), à «prend sa source» l'expression «prend sa source au flanc de ma première fiction ». Deux chemins descriptifs s'offrent alors à nous pour la compréhension de ce mécanisme d'interruption :

i. Il est tout d'abord possible de soutenir qu'il n'y a dans ces cas ni anaphore ni cataphore mais les deux à la fois. En ce sens, l'emphase (ou l'intensification), dans cette perspective, aurait pour portée tout aussi bien «faisait» (anaphoriquement) que « le tour complet de la mélancolie» (cataphoriquement) en (14) tout aussi bien «prend sa source» (anaphoriquement) que "au flanc de ma première fiction» (cataphoriquement) en (15). L'effet d'un tel découpage serait la mise en relief de l'originalité du choix de l'énonciateur dans la constitution d'une expression inédite et donc surprenante. Une telle interprétation, si elle nous semble juste dans ses constats (il s'agit d'anaphore \& de cataphore en même temps, d'où le mécanisme d'ana-cataphore) et dans ses conclusions (effet de surprise), elle nous paraît néanmoins très problématique dans son fonctionnement dans la mesure où nous pouvons difficilement continuer à parler de portée et d'intensification ou d'emphatisation puisque, d'une part, une seule portée s'appliquerait à deux termes (et non plus à un) et d'autre part, l'intensification ne s'exercerait au mieux qu'à des « morceaux » d'énoncé qui ne peuvent pourtant pas être accessibles à la compréhension individuellement. Nous l'aurons compris, le mécanisme d'ana-cataphore fait voler en éclats et la portée et l'intensification au sein de l'énoncé puisque l'énoncé n'est plus intelligible en soi.

ii. Nous pouvons secondement avancer que, dans l'ordre de la chaîne parlée, l'énonciateur commence à construire une expression qu'il sait d'emblée originale et potentiellement surprenante dans sa composition même. Puisqu'il la sait originale, il commence à la produire avec l'intention d'attirer l'attention. Dans ce cas, il interrompt le cours de son énoncé pour renforcer l'effet de surprise : dès lors, l'intensifieur disparait et devient un marqueur attentionnel. Nous ne sommes pas dans un mécanisme purement anaphorique ou purement cataphorique mais dans un mécanisme d'interruption dans la construction d'une tournure, qui se retrouve ainsi comme "suspendue » le temps d'une parenthèse située à l'échelle de la situation d'énonciation. L'effet de surprise dépend donc concomitamment de l'anaphore \& de la cataphore : c'est précisément l'interruption de la formulation à laquelle l'anaphore doit se rapporter qui provoque la curiosité de l'interlocuteur et qui attire son attention. La cataphore intervient comme pour profiter de cette attention afin que l'interlocuteur, à rebours, reconnaisse de facto l'audace sémantique de celui qui parle. Dans cette configuration, si je puis dire est le signe explicite d'une attitude entre deux locuteurs, dont l'un attire l'attention de l'autre. Cette attitude dépasse les mots et c'est précisément la raison pour laquelle la portée disparaît au profit de la relation. Toute cette séquence est assurée par un « jeu » anaphorique \& cataphorique, un jeu ana-cataphorique.

Au terme de ces descriptions, nous comprenons donc que si je puis dire intensifieur est majoritairement plus anaphorique, ce qui est sans doute dû à l'ordre de la chaîne parlée. Il n'en reste pas moins qu'il est également interprétable comme intensifieur cataphorique à part entière mais à la condition de s'appuyer sur des éléments qui, dans l'énoncé, rendent indiscutable ce rôle cataphorique. Dans le cas contraire, lorsque ni l'anaphore ni la cataphore ne sont décidables, la portée disparait, de même que l'intensification dans l'énoncé et nous passons à l'échelle de la situation d'énonciation où le MD vise à attirer l'attention de l'interlocuteur. Dès lors, il nous semble qu'au terme de cette analyse, nous pouvons établir une nouvelle distinction entre si je puis dire grammaticalisé et si je puis dire pragmaticalisé (c'est-à-dire MD à part entière) notamment en établissant une corrélation nette entre la position et la fonction. 


\subsection{La coexistence de deux si je puis dire: l'un grammaticalisé, l'autre pragmaticalisé}

En effet, il nous paraît désormais clair que, résolument cataphorique ou anaphorique, si je puis dire se situe davantage au stade de la grammaticalisation1 qu'à celui de la pragmaticalisation (grammaticalisation2) et que, quand il est ana-cataphorique, si je puis dire est clairement un MD (grammaticalisation2) qui n'intensifie plus quoi que ce soit dans l'énoncé lui-même mais devient le signe d'une relation entre deux locuteurs dans la situation d'énonciation. En somme, grammaticalisé, si je puis dire permet au langage de montrer sa propre audace en utilisant le locuteur (dans une démarche très métalinguistique) alors que si je puis dire pragmaticalisé (MD) permet au locuteur de montrer ses audaces en utilisant le langage (dans une démarche plus poétique).

La question de la dualité est ainsi clarifiée, pour ne pas dire stabilisée en français contemporain. Ainsi, lorsque si je puis dire est indiscutablement anaphorique ou indiscutablement cataphorique, son rôle porte sur l'énoncé a posteriori ou a priori, avec une nette disposition pour l'intensification de l'énoncé a posteriori. Dans ces deux configurations, le si je puis dire dont il est question reste à l'échelle de l'énoncé en assumant la fonction grammaticale d'emphase d'un élément de cet énoncé. On peut peut-être augurer dans cet emploi d'une certaine intention de l'énonciateur sur le plan illocutoire mais cela reste au stade de la supposition tant si je puis dire reste avant tout attaché au contexte de l'énoncé avec son rôle d'intensifieur.

Il en va autrement quand il vient attirer l'attention sur une expression en la disjoignant. L'effet produit affecte explicitement l'énoncé : il y a donc dans ce cas une intention manifeste (et expressive au sens de Dostie infra) qui ne peut être expliquée qu'au niveau de l'énonciation, c'est-à-dire au niveau du rapport entre l'énonciateur et le co-énonciateur. C'est ici la raison pour laquelle dans ce cas nous sommes en présence d'un autre si je puis dire, MD à part entière et plus particulièrement un « marqueur de réalisation d'un acte illocutoire » tel que décrit par G. Dostie (Dostie 2004 : 47) :

Ils ont la possibilité d'accomplir un acte illocutoire, le plus souvent expressif ou directif, parfois assertif. Il s'agit de mots-phrases ou de mots associés à un SN / une proposition traduisant l'état psychologique de l'énonciateur.

Comme nous le faisions remarquer supra, pour ce qui concerne si je puis dire MD, l'état psychologique dans lequel se trouve l'énonciateur relève de l'audace voire de l'aplomb et c'est la raison pour laquelle il intensifie l'expression qu'il introduit afin de ménager l'effet de surprise chez le co-énonciateur. Le mécanisme consiste en effet à isoler en les séparant les particularités sémantiques de chacun des termes à gauche comme à droite pour mieux souligner le caractère original voire la licence poétique de leur association inédite au vu précisément de leurs sémantismes si différents : c'est la puissance créatrice du locuteur qui s'exprime. Il en va ainsi en (14) et en (15) pour les expressions pour le moins non conventionnelles: «faire le tour complet de la mélancolie» \& «prendre sa source au flanc de ma première fiction » dans la mesure où l'on ne peut normalement pas faire le tour d'un sentiment ou prendre sa source au flanc. Or, l'état psychologiquede l'énonciateur (dont l'objectif est de faire remarquer une audace sémantique pour créer un effet de surprise) est paradoxal par rapport au choix des termes composant le MD. Ce dernier est effectivement composé par des termes typiques de la non actualisation, qu'il s'agisse de la mise sous condition avec si et la modalité avec pouvoir, autant d'éléments qui ne sont pourtant pas favorables à l'aplomb ou l'audace. Il s'agirait là d'une stratégie discursive qui consisterait à redoubler l'effet de surprise chez le co-énonciateur en lui attribuant, en plus de l'audace sémantique ellemême, dans l'énoncé, la validation cette l'audace, comme s'il pouvait peser sur la possibilité de sa production, dans la situation d'énonciation. Ce point vient achever de nous convaincre que tout mécanisme d'intensification ou de portée a bien disparu. Nous noterons, pour terminer la présente section que Si je puis dire tel que nous venons de le décrire fait partie d'un premier paradigme de MD commençant par $s i$ et exprimant justement une permission ou une possibilité feinte. 


\subsection{La variabilité du MD si je puis dire}

En français actuel (comme en moyen français avec si je puis comme décrit en 1.2.1), nous relevons également une variabilité de si je puis dire, notamment avec si je peux dire (forme similaire) et avec si je puis me permettre/si je puis m'exprimer ainsi/si je puis ainsi m'exprimer. Dès lors, cette variabilité paradigmatique conforte nos analyses menées supra en 2.2 sur l'interprétation à donner à si je puis dire MD comme une permission illocutoire.

\subsubsection{La variabilité si je puis dire / si je peux dire}

Pour comprendre la variabilité de ces deux MD si proches, nous avons soumis une nouvelle requête à la base Frantext : si je peux dire. Nous obtenons 51 résultats, soit 7,78 fois moins d'occurrences que si je puis dire. Un tel résultat peut s'expliquer par le fait que le MD si je peux dire apparaît tardivement (la première occurrence date de 1852 , la forme avec peux ayant sans doute été faite à partir de la forme contenant puis). Mais il ne s'agit pas, selon nous, de la seule explication. En effet, dans la mesure où, comme MD, si je puis dire est un marqueur d'appel à l'écoute, l'énonciateur le préférera à si je peux dire, expressivement moins fort que quand le marqueur est formé avec la singulière première personne puis. Cette question du rendement de puis apporte conséquemment une raison supplémentaire au maintien de la forme qui est à trouver à l'échelon pragmatique.

Cependant, si je peux dire remplit les mêmes fonctions que celles décrites en 2.2 pour si je puis dire. Ainsi, en (16) il est clairement anaphorique, clairement cataphorique en (17) et ana-cataphorique (donc MD) en (18) :

(16) Sur le moment, bouleversé par leur étrangeté, je n'ai pas eu la force de les regarder intellectuellement, si je peux dire (Paul Bourget, Le sens de la mort, 1915)

(17) Tu m'ennuies, tu m'inquiètes et, si je peux dire, tu me déçois. (Georges Duhamel, Chronique des Pasquier, 1934)

(18) Il n'est pas, si vous voulez, mon parent, mais mon allié plutôt, un allié, si je peux dire, par le sang. (Paul Claudel, Le soulier de satin, 1929)

A l'instar de si je puis dire, (16) fonctionne comme un intensifieur a posteriori, (17) comme un intensifieur a priori et (18) est un MD à part entière. De fait, si la dualité est commune à si je puis dire \& si je peux dire (grammaticalisés ou pragmaticalisés), il n'en est pas de même pour si je puis me permettre/si je puis m'exprimer ainsi/si je puis ainsi m'exprimer.

\subsubsection{La variabilité si je puis dire / si je puis me permettre / si je puis m'exprimer ainsi / si je puis ainsi m'exprimer ${ }^{16}$}

La variabilité paradigmatique s'établit en effet dans deux directions qui nous permettent de mieux discriminer encore les deux si je puis dire que nous décrivions supra :

i. D'une part si je puis dire varie avec si je puis m'exprimer ainsi \& si je puis ainsi m'exprimer ${ }^{17}$. Dans ce cas, la variation se fait avec si je puis dire grammaticalisé (dans grammaticalisation1) et la forme joue davantage le rôle grammatical de l'intensifieur préférentiellement anaphorique mais aussi cataphorique. En effet, la présence de l'unité «ainsi » ancre davantage le phrasème dans la référence à l'énoncé (a posteriori \& a priori) comme dans les exemples suivants :

(19) (...) tu aimais voir s'ébrouer Romann, et Nour, et Flo - le tiercé sur lequel tu avais tout misé ! Ô Misé Monde si je puis ainsi m'exprimer ! (Jean-Pierre Verheggen, Gisela, 2004)

(20) Pour y remédier, en renforçant si je puis m'exprimer ainsi, "l'équation personnelle » du futur Président, on peut penser qu'il faudrait qu'il soit choisi par la nation au suffrage universel (De Gaulle, Charles, Discours et messages. Conférence de presse tenue au Palais de l'Elysée le 11 avril 1961) 
Pour être complet sur cette première variabilité, nous noterons cependant que la dernière occurrence de $s i$ je puis m'exprimer ainsi date de 1982 et celle de si je puis ainsi m'exprimer de 2004. Cette remarque trouve son importance dans le fait que, paradoxalement, les occurrences de si je puis m'exprimer ainsi sont plus nombreuses mais moins récentes que celles de si je puis ainsi m'exprimer alors que la première variante est plus grammaticalisée (et sans nul doute beaucoup plus employée, encore de nos jours) que la seconde, plus rare, moins spontanée mais malgré tout maintenue et plus contemporaine. Ce paradoxe, selon nous, serait explicable par une coalescence moindre de si je puis m'exprimer ainsi \& de si je puis ainsi m'exprimer par rapport à si je puis dire, très coalescent et donc plus avancé dans la pragmaticalisation.

ii. D'autre part, si je puis dire varie avec si je puis me permettre. Dans cette configuration, nous quittons le paradigme de l'intensifieur dans l'énoncé et pour passer au paradigme du MD de la relation entre locuteurs comme en attestent les exemples à suivre :

(21) -Euh... si je puis me permettre. Vous savez, un garçon c'est bien aussi... Dans un lit, ça réchauffe... (Anna Gavalda, Ensemble c'est tout, 2004)

(22) Toujours aussi «siphonnée», si je puis me permettre (Régine Robin, Cybermigrances, traversées fugitives, 2004)

(23) Et même, je vous conseille à toutes les deux, de vous tenir tranquilles désormais. Cela dépasse vos moyens si je puis me permettre (Anne-Marie Garat, Dans la main du diable, 2006)

En (21), le MD marque une interruption du co-énonciateur pour l'introduction d'une objection. Il ne s'agit d'intensifier ni «Euh... » ni « Vous savez, un garçon, c'est bien aussi ». C'est, de manière très pragmatique, une interruption dans la situation d'énonciation (dans la relation entre l'énonciateur \& le coénonciateur). Les exemples (22) et (23) concernent davantage la manière dont l'énonciateur se considère lui-même dans l'acte d'énoncer, dans une sorte d'effet miroir. En effet, on pourrait vouloir interpréter $s i$ je puis me permettre en (22) comme étant l'intensifieur anaphorique de « siphonnée », d'autant que le terme est lui-même emphatisé par une mise entre guillemets. Or, selon nous, une telle approche serait faussée car si je puis me permettre ne concerne pas le terme employé en soi dans l'énoncé mais le fait d'oser l'employer tant il est familier. L'énonciateur signale par là même l'audace du choix du terme et n'intensifie pas l'unité mise entre guillemets. Au contraire, le MD, pragmatiquement, intervient comme un atténuateur ${ }^{18}$ (que l'on pourrait gloser par : j'emploie un terme familier, certes, mais $n$ 'allez pas croire que je le suis ou que je suis mal élevé). Nous relevons le même mécanisme dans (23), mais cette fois l'apaisement ne se fait pas sur un terme mais plutôt sur une situation entre les énonciateurs : ici, c'est la supériorité qui est affirmée et en même temps atténuée.

Nous déduisons de ces analyses qu'il existe un paradigme de MD dont la structure est $s i+j e+P 1$ pouvoir + verbe. Mais le paradigme ainsi décrit se limite-t-il à la seule construction autour du modal pouvoir ou rencontre-t-on d'autres MD construits autour d'autres modaux et commençant par si ?

\section{Conclusion : si je puis dire relève d'un paradigme plus large des MD commençant par Sl et contenant un modal ${ }^{19}$.}

\subsection{Le paradigme « large » SI+VMOD+(V)}

Le paradigme des MD commençant par si et contenant pouvoir à la première personne fait ainsi partie d'un autre paradigme, plus large : celui des MD commençant par si et contenant un modal dans la structure $\mathrm{SI}+\mathrm{VMOD}+(\mathrm{V})^{20}$.

En effet, nous regroupons dans cette catégorie tous les MD que nous venons de décrire, dans ce qui nous apparaît être maintenant un " paradigme second » construit autour de pouvoir à la P1, mais aussi des MD qui commencent également par si et qui comprennent l'autre modal vouloir, comme en (24), exemple extrait d'une réponse de Martin Hirsch pendant une émission de radio ${ }^{21}$ : 
(24) Martin Hirsch Moi j'ai de l'estime pour Xavier Darcos euh... et je ne sais pas s'il se sentait à l'aise à s'occuper des des des retraites ou du ministère des affaires sociales. Si vous voulez, moi mon opinion c'est que je trouve, on ne passe pas impunément d'un sujet à l'autre aussi éloigné que ça - trente ans sur les sujets d'éducation -, ce qui était Xavier Darcos, peut-être que c'est plus difficile dans d'autres domaines.

Dans ce paradigme émerge un nouveau couple modal, à l'échelle pragmatique, ie pouvoir et vouloir. Cette proximité pragmatique des deux modaux peut même se retrouver à proximité au sein de l'énoncé comme en atteste l'exemple (18) cité supra :

(18) Il n'est pas, si vous voulez, mon parent, mais mon allié plutôt, un allié, si je peux dire, par le sang. (Paul Claudel, Le soulier de satin, 1929)

Cet exemple est riche d'enseignements dans la mesure où il montre que le moyen par lequel le MD s'insère dans l'énoncé est le même aussi bien pour vouloir que pour pouvoir: l'interruption, la disjonction. En ce sens, si vous voulez scinde «il n'est pas mon parent» tout comme si je peux dire interrompt «allié par le sang». Ainsi, non seulement ce seul exemple met l'existence du paradigme « large » SI+VMOD+(V) en relief mais il vient révéler une similarité de fonctionnement du MD pour le couple modal pouvoir/vouloir. Dans le même temps, nous remarquons que vouloir se suffit à lui-même alors que si je peux a besoin de dire ${ }^{22}$.

\subsection{Les traits propres au paradigme SI+JE+POUVOIR+VERBE}

Nous noterons que ce paradigme a pour traits constants l'unité SI placée en tête et la présence d'un modal (pouvoir ou vouloir). Sont ainsi propres au « paradigme second» construit autour de pouvoir : la présence d'une première personne sous la forme puis ou peux (avec un rendement plus important pour puis) et celle d'un verbe obligatoire dans le co-texte droit. Le paradigme du MD construit avec pouvoir a donc besoin d'exprimer ce sur quoi s'exerce la modalité, qu'il s'agisse de l'énonciation (dire) ou des rapports entre énonciateur et co-énonciateur (permettre), avec une nette préférence pour l'énonciation (ie dire). Cette tendance particulière est confirmée par les statistiques relatives à la fréquence des co-textes gauche et droit de $p e u x^{23}$. La consultation de la base Wortschatz de l'Université de Leipzig indique en effet que dire se situe en troisième position parmi les occurrences les plus significatives du co-texte droit (significant right neighbours of peux) et je en première position parmi les occurrences les plus significatives du cotexte gauche (significantleftneighbours of peux). Nous en tirons deux conclusions :

i. la constitution d'un MD autour des trois seuls éléments $s i+j e+p u i s$ n'a pas survécu au Moyen-Français. ii. la proéminence, dans le MD, de la P1 et donc la forte présence de l'énonciateur est une caractéristique du MD paradigmatisé autour de pouvoir.

\subsection{Et « puis », alors?}

Nous relevions en 1 supra que :

i. le TLFi explique le maintien de la forme puis et par sa présence obligatoire en cas d'inversion du sujet, notamment dans les interrogatives, et par son emploi (non obligatoire du reste) en langue soutenue.

ii. le Grand Robert de la Langue Française qualifie la forme d'archaïque.

Certes, nous ne saurions remettre en cause les deux raisons syntaxique et de registre de i, cependant, nous admettrons dans le même temps que puis, même archaïque, continue à se développer.

En effet, comme nous venons de le souligner, puis se maintient aussi parce qu'il s'agit d'une unité qui est au cœur d'un ensemble grammaticalisé (a minima) ou en tant que MD à part entière : si je puis dire. La troisième raison qui explique le maintien de puis se trouve donc à l'échelle de la grammaticalisation ou de la pragmaticalisation. Si nous allons plus loin, nous pouvons même affirmer que, même s'il s'agit d'une forme ancienne (dont l'emploi est majoritairement sporadique sauf en cas d'inversion du sujet, selon la littérature), elle est une forme qui continue à se développer en français moderne et contemporain, très présente et très implantée (ce qui pose la question de l'emploi par nature exceptionnel en registre soutenu) car c'est assurément aux vingtième et vingt-et-unième siècles, en français moderne et 
contemporain, que son rendement grammatical ou pragmatique est le plus significatif, faisant du modal puis l'outil de l'expression intensifiée de l'audace ou de l'aplomb de l'énonciateur par excellence. Pour preuve, dans le cas du phrasème grammaticalisé comme dans celui du MD, puis sera préféré à peux, forme pourtant de facto (mais aussi a priori) plus moderne et plus fonctionnelle. Peut-être peut-on voir dans ce choix l'expression de la puissance d'un «je » énonciateur qui, ès qualité, mérite et réclame une forme, puis, qui ne ressemblera pas aux autres. Peut-être est-ce aussi la revendication de la puissance du dire qui s'actualise de facto en acte ${ }^{24}$ relationnel hardi de la part de l'énonciateur à destination du coénonciateur dans la situation d'énonciation, provoquant chez ce dernier une double surprise : celle de l'audace et de l'exclusivité sémantique telle qu'en train d'être énoncée et celle de l'illusion d'éventualité (le coénonciateur n'a en réalité pas le choix) dans la situation d'énonciation, l'énoncé étant de toute façon « verrouillé » par l'énonciateur. Une telle revendication expliquerait concomitamment la supériorité de $s i$ je puis dire sur ses variantes et l'ambiguïté de la forme quand il est intensifieur anaphorique, intensifieur cataphorique ou MD ana-cataphorique : dire dans l'énoncé ou dans l'énonciation ? Mais dans tous ces états, si je puis dire, c'est faire autorité. 


\section{Références bibliographiques}

\section{- Bases consultées}

Base Frantext (accès intégral) : www.frantext.fr

Wortschatz Universität Leipzig, corpus-based frenchdictionnary :

http://corpora.informatik.uni-leipzig.de/?dict=fra_mixed_2012

\section{- Composition du corpus d'étude}

Les exemples de notre étude sont issus de

L'interview de Martin Hirsch le 23 mars 2010 sur la radio RTL (retranscription intégrale)

Huit requêtes soumises à la base Frantext : si je puis, si je peux, si je puis dire, si je puis m'exprimer ainsi, si je puis parler ainsi, si je puis me permettre, si je puis ainsi parler, si je puis ainsi m'exprimer

\section{- Usuels et ouvrages généraux}

Le Grand Robert de la langue française, version « en ligne » entrée pouvoir : www.lerobert.com/espace-numerique/pro/le-grand-robert-de-la-langue-francaise

Le Trésor de la Langue Française informatisé, entrée pouvoir : http://atilf.atilf.fr/

Riegel, M., Pellat, J.-C. et Rioul, R. (2009). Grammaire méthodique du français. Paris : Presses Universitaires de France.

\section{- $\quad$ Etudes linguistiques}

Aptekman, J. (2006). « De la logique à la linguistique, une étude du sens de SI », doctorat en sciences du langage. Ecole des Hautes Etudes en Sciences Sociales (EHESS, Paris) et ENS (Paris)

Barbet, C et De Saussure L. (2012). Présentation: Modalité et évidentialité en français. Langue Française, numéro 173, Larousse, 3-12

Beeching, K. (2007). La co-variation des marqueurs discursifs bon, c'est-à-dire, enfin, hein, quand même, quoi et si vous voulez : une question d'identité ? Langue Française, numéro 154, 78 - 93

Charolles, M. et Fagard, B. (2012). En effet en français contemporain: de la confirmation à la justification/explication, Le français moderne, 2012 numéro 2, 171-197

Ciry, G. (2012). « Analyse multidimensionnelle du marqueur discursif « Si vous voulez » et de ses variantes. Master 2 Recherche en sciences du langage. Université de Valenciennes \& du Hainaut Cambrésis.

Dostie, G. (2004). Pragmaticalisation et marqueurs discursifs. Bruxelles : De Boeck, Duculot.

Dostie, G. et Pusch, CD. (2012). Présentation. Les marqueurs discursifs. Sens et variation. Langue Française, numéro 154, 3-12

Hopper, P.J. \& Traugott, E.C. (2003). Grammaticalization. Cambridge : Cambridge Textbooks in Linguistics, Second Edition.

Lamiroy, B. (1999). Auxiliaires, langues romanes et grammaticalisation, Langages, numéro 135, 33-45

Marchello-Nizia, C. (2006). Grammaticalisation et changement linguistique. Bruxelles : De Boeck, Duculot.

Paillard, D. (2011). Marqueurs discursifs et scène énonciative. In Hancil, S. (éd), Marqueurs discursifs et subjectivité. Rouen : Publications des Universités de Rouen et du Havre, 13-32

Pinto de lima, J. (2002). Grammaticalization, subjectification and the origin of phatic markers. In Wischer, I \& Diewald, G. (éd), New reflections on grammaticalization. Amsterdam: John Benjamins Publishing Company

Rossari, C. (1993). Les opérations de reformulation. Berne : Peter Lang

Rossari, C. (2012). Valeur évidentielle et/ou modale de faut croire, on dirait et paraît, Langue Française, numéro $173,65-81$

Siepmann, D. (2005). Discourse Markers Across Languages. New York : Routledge

Vanderbauwhede, G., Lauwers, P. \& Desmet, P. (2013). Les emplois référentiels du SN démonstratif en français. Essai de systématisation. Le français moderne, 2013 numéro 1, 17-41 


\footnotetext{
${ }^{1}$ Une autre requête sera proposée en 3 pour la description de la variabilité de Si je puis dire en Français contemporain.

${ }^{2}$ Il est d'emblée important de souligner que, même si les données qui ont servi à notre étude proviennent d'une base textuelle (et donc, stricto sensu, de l'écrit), les extraits produits et choisis revêtent une forte dimension orale et/ou conversationnelle. Pour preuve, (1) et (3) proviennent chacun d'une tragédie, (2) et (5) d'une comédie, et (4) d'une tragi-comédie d'origine espagnole. Les cinq premiers exemples « couvrent » donc un large spectre de genres théâtraux.

${ }^{3}$ Nous avons reproduit les résultats tels que livrés par la base Frantext. Nous choisissons de les exploiter tels quels. Cependant, nous devons attirer l'attention du lecteur sur le fait que l'auteur de la tragédie Alexandre n'est pas Jean de La Taille mais son frère, Jacques de La Taille.

${ }^{4}$ Le fait que (5) soit l'exemple le plus illocutoire n'a rien d'étonnant. En effet, Le Moyen de parvenir est un texte aux allures de farce composé à la manière des œuvres de Rabelais, ie reposant très largement sur le parler populaire et sur les bavardages grivois. C'est donc le plus oralisé des cinq exemples.

${ }^{5}$ Nous noterons qu'en dépit de son ambiguïté telle que décrite dans notre développement, (1) revêt d'emblée un aspect parenthétique dans la ponctuation même. Cet aspect sera caractéristique du MD en français moderne, au point d'introduire une interruption au sein de l'énoncé.
}

${ }^{6}$ La dimension parenthétique signalée dans la note 5 supra, notamment par la présence d'une forte ponctuation en (1) perdure certes de manière moins marquée (nous passons des parenthèses aux virgules) mais perdure tout de même en (2), (3), (4) et (5)

${ }^{7}$ Nous appelons « intensifieur » ou « emphatiseur » l'élément qui permet de construire une emphase telle que définie par la Grammaire méthodique du français (Riegel, Pellat, Rioul 2009 : 667) : "L'emphase - terme emprunté à la rhétorique - désigne tout procédé d'insistance ou de mise en relief. »

${ }^{8}$ Nous employons le terme de cataphore par opposition à celui d'anaphore (voir note 12 infra) et tel que décrit par la Grammaire méthodique du français (Riegel, Pellat, Rioul 2011: 1029) : "Conformément à son étymologie (anasignifie « en arrière », « en remontant»), le terme anaphore implique le renvoi à un élément antérieur du texte. Il s'oppose à cataphore, qui désigne le renvoi à un élément postérieur dans le texte (cata = «en bas », «en descendant »)»

${ }^{9} \mathrm{Il}$ est à noter que de récents travaux ont été consacrés au rapport jusqu'alors peu exploré entre les MD et la subjectivité en 2011 sous la direction de Sophie Ancil (cf. bibliographie).

${ }^{10}$ Parmi les étapes de constitution d'un MD, Gaétane Dostie décrit ainsi le processus de fixation de position : «La fixation de position: plus une unité se pragmaticalise, plus les éléments qui la composent deviennent fixes. Ce principe concerne les unités pragmatiques qui résultent de l'association de plusieurs unités lexicales/grammaticales, au départ libres. Certaines unités deviennent ainsi non déplaçables les unes par rapport aux autres, ce qui donne jour à un MD. Cette perte de liberté de position est en lien avec la coalescence. » (Dostie $2004: 36$ )

${ }^{11}$ De même qu'en note 10, Dostie décrit ainsi le phénomène de coalescence : « La coalescence : les unités adjacentes deviennent de plus en plus liées au fur et à mesure que la pragmaticalisation augmente. Ce principe est également axé sur les marqueurs formés par association de plusieurs unités, auparavant autonomes » (Dostie $2004: 36$ )

${ }^{12}$ Nous utilisons le terme anaphore par opposition à celui de cataphore défini en note 8 supra et tel que décrit par la Grammaire méthodique du français (Riegel, Pellat, Rioul 2009: 1029) : «L'anaphore se définit traditionnellement comme toute reprise d'un élément antérieur dans un texte (...) Plus précisément, une expression est anaphorique si son interprétation référentielle dépend nécessairement d'une autre expression qui figure dans le texte ». Pour si je puis dire, nous démontrons que pouvoir dire peut effectivement se rapporter à un référent antérieur dans l'énoncé

${ }^{13}$ La disposition particulière de cette troisième configuration ne nous permet plus de parler ni d'intensifieur ni de portée comme cela sera expliqué par la suite (voir notamment 3.1.3, 3.2 et la note 15 à ce propos).

${ }^{14}$ Nous relevons 12 occurrences pour le seul Philippe Lejeune, ce qui constitue une nouvelle illustration de la subjectivation décrite supra en 2.2.2. Le fait que le 'désir d'expressivité' s'affirme à ce point dans un ouvrage consacré à l'autobiographie ne doit, selon nous, rien au hasard.

${ }^{15}$ Comme signalé dans la partie 3, nous continuons à employer le terme d' « intensifieur » tant que l'élément sur lequel s'exerce la portée est indiscutablement identifiable au sein de l'énoncé, c'est-à-dire quand si je puis dire souligne un terme qu'il est possible d'identifier sans ambiguïté dans l'énoncé. Il est clair qu'il ne peut plus être considéré comme un intensifieur et avoir une portée lorsqu'il est MD car, dans cette configuration, il passe au plan relationnel entre deux locuteurs et est ancré cette fois dans la situation d'énonciation. La linéarité nécessaire au couple intensifieur/portée est brisée. En tant que MD, son rôle est d'attirer l'attention sur un fait langagier audacieux revendiqué comme auprès de l'interlocuteur de la part de celui qui parle.

${ }^{16}$ Le manque de place ne nous permettra pas de développer ce point ici, mais il serait bien évidemment tout aussi intéressant de comparer la variabilité paradigmatique de puis telle que décrite ici avec la variabilité paradigmatique de peux, notamment en nous intéressant aux phrasèmes si je peux dire / si je peux me permettre / si je peux employer ce terme / si je peux ainsi m'exprimer. Nous avons soumis ces requêtes à la base Frantext et avons déjà quelques pistes d'analyse. 
${ }^{17}$ La description que nous proposons de si je peux m'exprimer ainsi \& de si je peux ainsi m'exprimer est aussi valable pour si je puis parler ainsi \& si je puis ainsi parler car ces deux variantes sont similaires dans leur fonctionnement et quasi égales dans le nombre de leurs occurrences.

${ }^{18} \mathrm{C}$ 'est du reste dans ce rôle qu'Aptekman décrit si je puis dire : « Il s'agit donc ici de formuler une réserve sur un terme employé, qui permet d'en atténuer la portée. On remarque que de telles valeurs sont fortement associées à des formes typiques (SI l'on veut, SI je puis dire, SI l'on peut parler de...) » (Aptekman $2006: 166$ )

${ }^{19}$ La citation présentée dans la note précédente (note 18) recense le paradigme dont il est question ici.

${ }^{20}$ Dans ce paradigme "plus large », le verbe se trouvant dans le co-texte droit du modal devient effectivement optionnel.

${ }^{21}$ Extrait de la retranscription intégrale de l'interview de Martin Hirsch le 23 mars 2010 sur la radio RTL (retranscription faite par l'auteur de ces lignes)

${ }^{22}$ Nous n'avons pas le temps de développer ce point ici, mais la comparaison vouloir+ensemble vide et pouvoir + dire mérite de faire l'objet d'une étude approfondie.

${ }^{23}$ Nous n'avons pu soumettre au Wortschatz de l'Université de Leipzig que la requête «peux » dans la mesure où la requête " puis » ne donne pas accès à la $\mathrm{P} 1$ de pouvoir mais à la conjonction puis

${ }^{24}$ Il est intéressant de noter que le verbe «faire» est bien placé parmi les cooccurrences significatives du co-texte droit de peux selon le corpus de Leipzig puisqu'il apparaît en sixième position. C'est du reste le deuxième verbe le plus significatif dans le co-texte droit après dire. 